\title{
Ein Kommentar zum Kommentar der Authentizität. Zur koreanischen Übersetzung der Lebensläufe von Alexander Kluge
}

Un commentaire sur le commentaire de l'authenticité. À propos de la traduction coréenne des Lebensläufe d'Alexander Kluge

A commentary on the commentary on authenticity. The korean translation of Alexander Kluge's Lebensläufe

\section{Hosung Lee}

\section{OpenEdition}

Journals

Édition électronique

URL : http://journals.openedition.org/ceg/1190

DOI : $10.4000 /$ ceg. 1190

ISSN : 2605-8359

Éditeur

Presses Universitaires de Provence

Édition imprimée

Date de publication : 18 décembre 2015

Pagination : 141-152

ISBN : 979-1-03200-020-5

ISSN : 0751-4239

\section{Référence électronique}

Hosung Lee, « Ein Kommentar zum Kommentar der Authentizität. Zur koreanischen Übersetzung der Lebensläufe von Alexander Kluge », Cahiers d'Études Germaniques [Online], 69 | 2015, Online erschienen am: 17 Dezember 2017, abgerufen am 26 November 2020. URL : http://journals.openedition.org/ceg/ 1190 ; DOI : https://doi.org/10.4000/ceg.1190 


\title{
Kommentar zum Kommentar der Authentizität. Zur koreanischen Übersetzung der Lebensläufe von Alexander Kluge
}

Hosung LEE

Freie Universität Berlin

\begin{abstract}
Wer weiß, wer wei $\beta$, ob der Intellektuelle nicht durch eine unerbittliche Bestimmung und gegen seinen Gefallen oder Willen dazu berufen worden ist, in dieser Welt Paradoxie zu vertreten! ${ }^{1}$
\end{abstract}

I.

Die koreanische Übersetzung der erstmals 1962 erschienenen Kurzgeschichtensammlung Lebensläufe von Alexander Kluge ist im Dezember 2012 in der Reihe „Weltliteratur“ im Verlag Eulyoo in Seoul veröffentlicht worden. ${ }^{2}$ Als Grundlage diente die Suhrkamp-Ausgabe von 1986, eine der erweiterten Versionen. ${ }^{3}$ Als Übersetzer dieses Werks möchte ich nicht nur über die Schwierigkeiten, auf die man bei der Übersetzung der Klugeschen Werke trifft, sondern auch über die kooperative Autorschaft Kluges im allgemeinen referieren.

Obwohl es schon mehrmals Retrospektiven seiner filmischen Werke in Korea gab, ist Alexander Kluge kein Autor, dessen Literatur in Ostasien bevorzugt übersetzt worden wäre. Im Vergleich $\mathrm{zu}$ anderen zeitgenössischen Autoren aus dem deutschsprachigen Raum oder auch zu seinen eigenen Filmen wurde Alexander Kluges literarischen Werken in Ostasien bisher nur wenig Beachtung geschenkt. Die Übersetzung ist der Versuch, erstmalig eines seiner selbständigen literarischen Werke zu übertragen. Im Gegensatz dazu wurde die erste englische Übersetzung dieses Werks bereits im Jahr 1966 von Leila Vennewitz erstellt. ${ }^{4}$

1 José Ortega y Gasset, Miseria y Esplendor de la Traducción/Elend und Glanz der Übersetzung, übers. v. Gustav Kilpper, München, Langewiesche-Brandt, 1956, S. 42.

2 알렉산더 클루게, 이력서들, 이호성 옮김, 서울, 을유문화사, 2012 (Alexander Kluge, Lebensläufe, übers. v. Hosung Lee, Seoul, Eulyoo Publishing Co., 2012), im Folgenden zitiert als ÜLL.

3 Alexander Kluge, Lebensläufe, Frankfurt a. M., Suhrkamp, 1986, im Folgenden zitiert als LL.

4 Alexander Kluge, Attendance List for a Funeral, übers. v. Leila Vennewitz, New York/London/ Toronto/Sydney, McGraw-Hill Book Company, 1966; Alexander Kluge, Case Histories, übers. v. Leila 
Einer der Gründe für die spät erfolgte Übersetzung besteht vermutlich darin, dass sich der Autor seit den achtziger Jahren bis zum Jahr 2000 hauptsächlich mit Fernseharbeiten beschäftigt hat. Daneben sind die verborgenen, manchmal verschwiegenen Fakten und die Lücken in Rechnung zu stellen, die Kluges Werke enthalten und interpretationsbedürftig sind, besonders wenn sie dem Publikum eines anderen Kulturraums vorgestellt werden sollen. Schließlich sollten auch die Mehrstimmigkeit und die Mehrdeutigkeit der Klugeschen Sprache, die die Leser scheinbar oft in die Irre führen und verwirren, als Grund angeführt werden, warum keine Übersetzung früher erschien. Mit der einseitigen Entscheidung über die Bedeutung von Wörtern, Sätzen und ganzen Passagen könnte sich der Übersetzer dem Vorwurf mangelnder Umsicht aussetzen. Alle genannten Aspekte haben mit Kluges kompliziertem Konzept der Bildung zu tun, das seinerseits nicht leicht zu verallgemeinern und zu übertragen ist.

\section{II.}

Kluge versieht die Tätigkeit des Philologen mit bedenklichen Geschichten von Vertretern dieses Standes - wie z. B. „E. Schincke“, „Ein Berufswechsel“, „Der Pädagoge von Klopau“ - und stellt sie gerade in seinem ersten Werk Lebensläufe recht ironisch dar. Trotzdem war es nötig, die Übersetzung unter Beachtung philologischer Tugenden durchzuführen. Denn wenn man als Übersetzer das Ziel verfolgt, den Originaltext seinen fremdkulturellen (und oft seinen heimischen) Lesern authentisch zu vermitteln, muss man als der treueste, aktivste und gelegentlich pedantischste Leser und Philologe diverse Inter- und Kontexte der Worte, Zitate und der Geschichte aufspüren.

In den Lebensläufen gibt es mehrere Erzählungen von gescheiterten Gelehrten. Der Altphilologe E. Schincke in dem gleichnamigen Text lässt sich anfangs von Hitler täuschen und betrachtet die nationalsozialistische Bewegung als zweite Romantik. Am Ende aber versteckt er seine Schüler in einer abgelegenen Scheune, um sie vor der Kriegssituation und der Rekrutierung zum Militäreinsatz zu schützen. Dort unterrichtet er die Schüler und schreibt über die frühere deutsche Bildungsgeschichte. Hier ein Auszug der mit Latein gemischten deutschen Sätze: „Eruditio bedeutet demnach inhaltlich einmal: fidei rationes (praecepta dei) scire und diese sobria conversatione ostendere (ea implere). Der ductor et doctor gregis führt docendo et ammonendo zur salus animarum. Das geht alle an: docendus est itaque (omnis) homo rationalem habens intelligentiam". 5

Diese Art von nicht bzw. halb übersetzten Erklärungen der Bildungsidee des karolingischen Geistlichen und Wissenschaftlers Alkuin erstrecken sich über mehr als fünf Seiten. Im Gegensatz zu der englischen Übersetzung, in der der lateinische Text nicht vollständig enthalten ist und nicht erläutert wird, habe ich ihn bei der

Vennewitz, New York/London, Holmes \& Meier, 1988.

5 LL, S. 86. Dieser Teil fehlt in der englischen Fassung von Vennewitz. 
koreanischen Übersetzung auf Latein wiedergegeben und in Klammern mit der koreanischen Übersetzung versehen, wie die folgenden Sätze:

\begin{abstract}
따라서 eruditio(교양)이란 내용적으로 일단 이런 뜻이다. fidei rationes (praecepta dei) scire(믿음의 근거 - 신의 명령 - 를 아는 것이고), 또 이렇게 sobria conversatione ostendere (ea implere)(올바른 삶의 태도를 보여주는 것이다 - 의무 수행). 이 ductor et doctor gregis(민중의 영도자이자 선생)은 docendo et ammonendo(수업과 훈계를 통해) salus animarum(영혼의 치유)를 수행한다. 이는 사람이라면 누구에게나 해당하는 것이다. 그러므로 docendus est itaque (omnis) homo rationalem habens intelligentiam(지성을 가진 이성적인 - 모든 - 인간은 배우는 것이다. ${ }^{6}$
\end{abstract}

Auf Deutsch besagen diese Sätze, dass Bildung bedeutet, die Gründe des Glaubens, nämlich die Gebote Gottes zu kennen und durch Erfüllung der Gebote eine nüchterne Lebensweise zu zeigen. Der Führer und Lehrer des Volkes führt durch Unterricht und Mahnung die Seelen zum Heil. Dies gehe die Menschen qua Vernunftbegabung an, so dass allen die Weisheit gelehrt werden muss.

Es werden hier zwei historische Situationen parallelisiert, die sich auf Bildungskonzepte und die damit verbundene politische Macht beziehen. Zuerst wird das Wort Führer (ductor) mit Lehrer (doctor) verbunden und somit auch die Herrschaftsform der Karolingerzeit mit deren Bildungsidee. ${ }^{7}$ Zugleich werden damit Konzepte zweier entfernter Zeiten, nämlich des Reichs von Karl dem Großen und des Dritten Reichs nebeneinandergestellt. Im Zusammenhang mit dem oben angeführten Zitat erfährt der Leser von einer Geschichte, in der der Geistliche Alkuin versucht, einen Schüler, der einen Fehler begangen hat, vor Karl dem Großen und dem Bischof Theodulf zu schützen und ihn in einer Kirche zu verstecken. Analog entwickelt sich die Geschichte von Schincke, der seine Schüler vor den Nationalsozialisten verbergen will. Schincke hat am Ende jedoch keinen Erfolg, da ihn die Nationalsozialisten entdecken und seine Schüler mitnehmen. Das Ende von Alkuins Geschichte lässt Kluge offen.

Die beiden Geschichten von Schincke und Alkuin werden ohne jegliche Übersetzung in doppelter Weise parallel erzählt. Weder gibt es in der Geschichte „E. Schincke“ eine Übersetzung des Lateinischen noch gibt es eine direkte „Übersetzung“ der alten Geschichte aus der Karolingerzeit in die neuere des Dritten Reichs. Es ist nicht ganz klar, ob der Erzähler oder die Hauptfigur die mittelalterliche mit der nationalsozialistischen Geschichte inhaltlich verbindet. Bei Kluges montierten Texten bleibt es oft vage, ob das Subjekt der Aussage der Erzähler, eine Figur oder der Autor selbst ist. Diejenigen, die diese Parallelwelten ineinander ,übersetzen“, sind die aktiven Leser, welche die Geschichten lesen, interpretieren und Verknüpfungen vornehmen. Der Text enthält gewissermaßen eine Botschaft an seine Ausleger, nämlich Philologen oder Übersetzer, und ist insofern selbstreflexiv.

Die vielen Tippfehler und falschen Quellenangaben, die in Kluges Zitaten zu verzeichnen sind, blieben bis zur Chronik der Gefühle im Jahr 2000 durch mehrmalige Veröffentlichungen der Lebensläufe hindurch unverändert, was die Übersetzung der lateinischen Texte in der Praxis schwer machte. ${ }^{8}$

6 ÜLL, S. 103.

7 LL, S. 87.

8 Auf den Buchstabenwechsel zwischen $\mathrm{C}$ und $\mathrm{K}$ in dem Namen von Alcuin (Alkuin) in der Geschichte und bei den Zitaten sollte auch hingewiesen werden. 
Die Hauptfigur Schincke liest am Anfang der Geschichte das Buch Deutscher Geist in Gefahr (1932) von Ernst Robert Curtius und glaubt in der nationalsozialistischen Bewegung einen idealen Kern zu sehen, den der Verfasser des Buches keineswegs sah. Die beiden (d. h. die durch Tippfehler verursachte Schwierigkeit und die falsch interpretierende Figur) ließen sich als eine Mahnung an die Philologen hinsichtlich der Unmöglichkeit einer Übersetzung oder Interpretation verstehen.

Einerseits wegen des Auftrags, dass der Übersetzer im Prinzip auch alle Fremdtexte, die der Autor versteckt hat, verstehen und übersetzen soll, andererseits aus Neugier, die der heimliche Zusammenhang der Geschichte hervorruft, wird der Übersetzer dazu gezwungen, auf ,die Absicht des Autors“ aufmerksam zu werden. ${ }^{9}$

Damit würde man in diesem Fall auf eine andere Entdeckung und einen anderen Zusammenhang kommen. Teile der Alkuin-Geschichte und der Kommentare sind unverändert aus dem Buch Eruditio und sapientia: Weltbild und Erziehung in der Karolingerzeit. Untersuchungen $z u$ Alcuins Briefen ${ }^{10}$ von Wolfgang Edelstein übernommen worden, das erst im Jahr 1965 veröffentlicht wurde, während Kluges Lebensläufe bereits 1962 erschienen.

Die Fehler sind in dem mutmaßlichen Originaltext von Edelstein nicht vorzufinden. Man fragt sich demnach, ob es sich in Kluges Text um absichtliche Fehler handelt, die das Konzept der reinen und genuinen Autorschaft in Zweifel ziehen. Man kann mutmaßen, dass Kluge Einsicht in das unveröffentlichte Manuskript Edelsteins noch vor der Veröffentlichung hatte oder aber Edelstein die Idee Kluges weiterentwickelte. Edelstein und Kluge waren Ende der fünfziger Jahre Mitarbeiter in der Kanzlei von Hellmut Becker, „der in den sechziger und siebziger Jahren als ,heimlicher Bundeskultusminister ${ }^{6}$ galt ${ }^{\text {" }}{ }^{11}$ Eine Form der Kooperation und Kritik liegt jedenfalls nahe. Bereits in Lebensläufe zitierte Kluge die Idee Hellmut Beckers über die Freiheit des Lehrers in der Geschichte „Berufswechsel “, und noch heute erzählt er in seinen jüngsten Veröffentlichungen wie Das Fünfte Buch - Neue Lebensläufe von den Erfahrungen mit Hellmut Becker sowie anderen Intellektuellen der alten Bundesrepublik. ${ }^{12}$

Die menschlichen und persönlichen Beziehungen zwischen den Intellektuellen, die sich mit der realen Bildungspolitik in der deutschen Nachkriegszeit beschäftigt haben, und deren Wirkung auf ihre Texte ziehen immer mehr Aufmerksamkeit auf sich, wenn man den Text mit Lücken in die andere Sprache kohärent zu übersetzen versucht.

\footnotetext{
9 Vgl. Willam K. Wimsatt/Monroe C. Beardsley, ,The Intentional Fallacy“, in The Sewanee Review 54, Sewanee, 1946, S. 486ff. In diesem Artikel wurde über T. S. Eliots Gedicht The Love Song of J. Alfred Prufrock und dessen vage intertextuelle Referenz zu einer Szene aus einem Gedicht von John Donne diskutiert.

${ }^{10}$ Wolfgang Edelstein, Eruditio und sapientia: Weltbild und Erziehung in der Karolingerzeit. Untersuchungen zu Alcuins Briefen, Freiburg, Rombach, 1965.

${ }^{11}$ Vgl. Jan-Martin Wiarda, „Hellmut Becker ,Der Strippenzieher““, in Die Zeit, Nr. 21 vom 16. Mai 2013. Sie entwarfen ein Konzept für ein neu zu gründendes Max-Planck-Institut für Bildungsforschung. Becker wurde Gründungsdirektor, Edelstein erster wissenschaftlicher Mitarbeiter des Instituts und später auch Direktor in Berlin. Vgl. Heike Schmoll, „,Demokratie lernen - reformpädagogisch“, in Frankfurter Allgemeine Zeitung, Nr. 66 vom 19. März 2010, S. 10; Reinhard Kahl, „Wurzeln und Flügel. Der MPIBildungsforscher Wolfgang Edelstein wird 75“, in Süddeutsche Zeitung, Nr. 134 vom 14. Juni 2004, S. 9; Reinhard Kahl, „Lehrer, Forscher, Berater: Porträt eines Rastlosen“, in Die Zeit, Nr. 20 vom 8. Mai 2002.

12 Vgl. LL, S. 156; Alexander Kluge, Das Fünfte Buch. Neue Lebensläufe. 402 Geschichten, Berlin, Suhrkamp, 2012, S. 104f.
} 
Als koreanischer Übersetzer und Philologe wollte ich die Überschneidung der drei historischen Ebenen deutscher Bildungsgeschichte neu entdecken und sie den Lesern der Übersetzung erläutern.

\section{III.}

Hinter den persönlichen Lebensläufen seiner Figuren verbirgt der Autor historische und chronologische Zusammenhänge, die der Verständlichkeit halber vom Übersetzer zumindest in seinen Kommentaren zum Vorschein gebracht werden müssen. Darüber hinaus gibt es dokumentarische, faktische Tatsachen, die Kluge häufig ein wenig verändert und verfremdet, die aber trotzdem noch authentisch wirken. Als Beispiel wird hier die Geschichte von „Oberleutnant Boulanger“ angeführt werden. Der Offizier dieser Erzählung hat die Aufgabe „die jüdisch-bolschewistischen Kommissare“"13 für die Rassenforschung von den übrigen Gefangenen zu „selektieren“, danach zu enthaupten und die Köpfe in Konservierungsbehältern an die Reichsuniversität Straßburg zu versenden.

\section{Die Aufgabe, Präzision in das hoffnungslose Ende der kriegsgefangenen Kommissare zu bringen, erfüllte B. im Sommer und Winter 1942 in den Bereichen der Heeresgruppen Mitte und Süd (später der beiden südlichen Heeresgruppen). Im Februar 1943 wurde sein Tätigkeitsbereich auf den Kreis der Heeresgruppe Mitte begrenzt. ${ }^{14}$}

Die Datierung und die Ortsangaben weisen darauf hin, dass der Leser auf den $\mathrm{Zu}$ sammenhang aufmerksam gemacht werden soll. Es geht in diesem Zeitraum um die „Schlacht von Stalingrad“, mit der sich Kluge in seinem zweiten Buch Schlachtbeschreibung ${ }^{15}$ auseinandergesetzt hat. Nur mit den beiden zitierten Sätzen parallelisiert der Erzähler die Taten des Oberleutnants Boulanger mit der Schlacht, die 1943 mehr als eine halbe Million Menschenleben gekostet und die Lage des Krieges zuungunsten des Deutschen Reiches verändert hat. Wenn ein Leser ohne Erläuterung solcher Punkte den Text weiterlesen würde, könnte er diesen Abschnitt für belanglos halten.

Die Beschreibung klingt authentisch - wenn wir die Aussage oben als Boulangers eigene verstehen würden -, nicht nur weil er als Individuum die gesamte historische Situation nicht selbst erfahren könnte, sondern auch weil sich seine Sinne vor den grausamen Tatsachen schützen würden. Es ist jedoch die Aufgabe des aktiven Lesers, solche Tatsachen wiederzuentdecken. Kluge sagt in seiner Adorno-Preis-Rede:

Es ist Verknüpfungsarbeit (und wenn eine Weberin ihrer Arbeit nachgeht, nennt man das Text) notwendig, um das Nebeneinander von Rettung und Verhängnis wahrzunehmen, die Heterotopie. Man muss das Allgemeine, das Besondere und die tückische Einzelheit drehen und wenden, wie es die Spinnerin Arachne bei Ovid mit ihren Netzen tut. Man muss die Fakten zu einer Erzählung zusammenfügen. Erlöst die Fakten von der menschlichen Gleichgültigkeit! ${ }^{16}$

13 LL, S. 11.

14 Ibid., S. 17.

15 Alexander Kluge, Schlachtbeschreibung, Olten/Freiburg im Breisgau, Walter-Verlag, 1964.

16 Alexander Kluge, „Die Aktualität Adornos. Rede zum Theodor-W.-Adorno-Preis 2009“, in Personen und Reden, Berlin, Wagenbach, 2012, S. 69. 
Unter den zahlreichen beachtenswerten Punkten in den Lebensläufen ist noch ein weiteres Beispiel anzuführen. Das folgende Zitat ist in die Kurzgeschichte „Abbau eines Verbrechens durch Kooperation“ eingefügt.

Bertrand Russel, Power, Zürich 1947, S. 214: „So muß es zwei Polizeikörper und zwei Scotland Yards geben, von denen der eine, wie es heute der Fall ist, die Schuld, der andere die Unschuld nachweisen muß; so muß es zwei Erfahrungsweisen von Verbrechern geben: die einen, die es aufbauen, die anderen, die es wieder abbauen“. ${ }^{17}$

Die Übersetzung von Bertrand Russells Power $^{18}$ ist 1947 unter dem Titel Macht ${ }^{19}$ in Zürich erschienen. Die Übersetzung enthält das Zitat allerdings nicht auf Seite 214, sondern auf Seite 237. In der Originalausgabe steht es auf Seite 296. Schon Kluges „Tippfehler“ im Namen eines der bekanntesten Philosophen der Welt - Russel statt Russell - ist kaum zu übersehen. Wichtiger aber ist es darauf hinzuweisen, dass nur der erste Teil dieses Zitates von Russell stammt. Der zweite stammt von Kluge, er versteckt sich somit in dem Zitat eines anderen. Die Frage, ob Kluge hier den Gedanken Russells in seiner eigenen Weise entwickelnd weiterführt oder ob er einfach einen Gedanken gegen Russell in das Zitat einflicht, hängt davon ab, wie man den „Kommentar“ Kluges im Kontext der Geschichte „Abbau eines Verbrechens" und der Lebensläufe interpretiert. Es handelt sich in dieser Geschichte um die Wiederbelebung eines Toten durch die sorgsame Pflege und die Gefühle einer Prostituierten. Im 16. Kapitel der Übersetzung Macht, in dem sich die Seite 214 befindet, geht es um die Machtliebe der Philosophie. Russell versucht zu beweisen, dass fast alle Richtungen der Philosophie von der Liebe zur Macht abstammen, und er zieht die Schlussfolgerung: „Wenn das gesellschaftliche Leben gesellschaftliche Wünsche befriedigen soll, muß es sich auf eine Philosophie stützen, die nicht aus der Machtliebe abgeleitet ist" ${ }^{\text {"20 }}$. Auf Seite 214 interpretiert Russell, wie sich die Hegelsche Philosophie über die Wahrheit der Geschichte und Literatur äußert: „[...] es gibt tatsächlich keinen Unterschied zwischen Wahrheit für den Novellisten und Wahrheit für den Historiker. Das gibt der schöpferischen Phantasie Freiheit - die Phantasie wird von den Fesseln einer angenommenen ,realen' Welt befreit." ${ }^{21}$ Daneben gibt es Russells Interpretation von Pragmatismus und Bergsons Lebensphilosophie, dass sie auch Machtliebe seien. Russell kritisiert die Passivität der Intellektuellen in Bezug auf die beiden Philosophien.

Bei Kluges Texten fließen nicht nur die Fakten und die Fiktionen zusammen, sondern auch die Stimmen von Autoren, Erzählern und Figuren ineinander. Die vermischten Stimmen verschiedener Sprecher in Kluges Geschichten, deren Träger bei der Übersetzung - schon wegen der Grammatik ostasiatischer Zielsprachen mehr oder weniger deutlich gemacht werden sollten, fordern den Übersetzer zu

${ }^{17}$ LL, S. 248.

${ }^{18}$ Bertrand Russell, Power: A New Social Analysis, London, George Allen \& Unwin, 1938.

19 Bertrand Russell, Macht. Eine sozialkritische Studie, übers. v. Stephan Hermlin, Zürich, Europa Verlag, 1947.

20 Ibid., S. 218

${ }^{21}$ Ibid., S. 214. 
einer Interpretation und Auseinandersetzung heraus, die sich in der Umgestaltung des Originaltexts um ihre eigene Authentizität bemühen muss.

\section{IV.}

Aus der Frage, wie man den Klugeschen Text authentisch übersetzen kann, ergibt sich die Folgefrage, warum der Autor sich selbst als bloßen Sammler und Chronisten bezeichnet. Adorno zufolge darf man weder das Werk mit der Absicht des Autors verwechseln, noch „den Dichter, überästhetisch, als Stifter, ohne das Agens der Form konkret zu reflektieren [verherrlichen]“22. Diese Gebote werden legitimiert, gleichzeitig aber ironisiert, wenn man an die Zusammenarbeit von Alexander Kluge mit Philosophen, Wissenschaftlern, Schriftstellern und Künstlern denkt. Sowohl die Zitate aus den fiktiven Quellen, als auch die aus den Apokryphen, die aus noch nicht veröffentlichten Texten von Mitarbeitern stammen, wirken authentisch. Vor allem weil es andere Schriftsysteme als die wissenschaftlichen Schriftsysteme gibt und die ersten die letzteren, denen man leicht Glaubwürdigkeit zuschreibt, nachahmen. Die Authentizität bei Kluge ist aber anders als jene Objektivität bei der Wissenschaft. Bei Kluges Texten treffen die verschiedenen Schriftarten aufeinander und kooperieren oder konkurrieren um Authentizität.

Harro Müller vergleicht die Verwendungsweisen des Authentizitätsbegriffs bei Theodor W. Adorno und Alexander Kluge ${ }^{23}$ und versucht, Adornos Begriff von Authentizität durch den von Kluge zu korrigieren. Adorno sagt: „Je authentischer die Werke desto mehr folgen sie einem objektiv Geforderten, der Stimmigkeit der Sache, und sie ist stets allgemein." ${ }^{24}$ Deshalb deutet Müller Adornos Konzept der Authentizität dahingehend, dass es „positiv“, „komparativ“ und „superlativ“" sei. ${ }^{25}$ Kluge sagt: „Authentizität heißt: daß eine Situation stimmig ist, nicht bloß, daß ein Sachverhalt oder die Formen stimmen. Authentisch ist auch die direkte Konfrontation von kollektivem Umfeld und Individualität, die nicht gegeneinander verwischt werden, sondern auch einen Moment der Produktion von Individualität beinhalten“. ${ }^{26}$

Kluge zufolge ist Authentizität also nicht ein Produkt der objektiven Autorität, sondern der Situation. Hierbei ließe sich an die Beziehung zwischen Original und Übersetzung denken. Dabei geht es nicht nur um die Treue gegenüber dem Originaltext oder die Freiheit bei der Übersetzung. Wenn man die Worte Kluges auf die Authentizität der Übersetzung anwenden sollte, könnte man sie so verstehen,

${ }^{22}$ Theodor W. Adorno, „Parataxis“, in Noten zur Literatur, Gesammelte Schriften, Bd. 11, Frankfurt a. M., Suhrkamp, 2003, S. 452. Es ist eine Kritik Adornos an Heideggers Hölderlin-Interpretation und Hölderlin-Verehrung.

${ }^{23}$ Harro Müller, „Verwendungsweisen des Authentizitätsbegriffs bei Theodor W. Adorno und Alexander Kluge“, in Christian Schulte (Hrsg.), Die Frage des Zusammenhangs. Alexander Kluge im Kontext, Berlin, Vorwerk 8, 2012, S. 50ff.

${ }^{24}$ Theodor W. Adorno, Ästhetische Theorie, in Gesammelte Schriften, Bd. 7, Frankfurt a. M., Suhrkamp, 1970, S. 300.

${ }^{25}$ Müller, „Verwendungsweisen“, S. 51.

${ }^{26}$ Alexander Kluge, Bestandsaufnahme: Utopie Film, Frankfurt a. M., Zweitausendeins, 1983, S. 214. 
dass der Übersetzer sich über sein subjektives Gefühl und sein kooperatives Verhältnis gegenüber der Situation des Buchmarktes, den Regeln des Verlags und der akademischen Situation klar werden muss. Kluge betont immer wieder, man schreibe Bücher nicht allein. ${ }^{27}$ Die Auswahl eines Werkes aus einer fremdsprachigen Literatur zur Übersetzung hängt einerseits davon ab, wie weit die gesellschaftliche Situation des Originaltextes mit der Ausgangskultur zu verbinden ist. Andererseits hat sie auch damit zu tun, mit wem der Übersetzer zusammenarbeitet und in welcher Umgebung er übersetzt. Dem Übersetzer stellt sich immer wieder die Frage, ob „eine Situation stimmig ist“. Die vielseitigen Widersprüche der „Gesellschaft“ und ihre kooperativen Verhältnisse bleiben immer Kontext der Literatur und ihrer Übersetzung.

Ich möchte nicht behaupten, dass der Übersetzer gleichberechtigt neben dem Autor steht, wie jüngere Forschungen zu beweisen suchen ${ }^{28}$. Es soll hier nur betont werden, dass die Klugesche Autorschaft selbst auch von verschiedenen Schrifttätigkeiten beeinflusst wird und dass seine Haltung wiederum ein Vorbild für Übersetzer werden könnte. Man betrachte Kluges Gedanken über die „Transkribisten“:

Eine Transkription der Texte (so wie schon die Evolution an ihren DNA-Texten bastelte) schafft nicht nur Linien zu künftigen neuen Texten. Sie ist auch als Rekonstruktion möglich in Richtung des Paradieses. Der Weg dorthin führt über Unschärfen. „Näher, mein Gott, zu Dir", das war das Musikstück, das die Bordkapelle der Titanic noch bis zuletzt gespielt hatte. Es ist aber auch die Arbeitsanweisung an die Transkribisten aller Länder, welche vom Erdmittelpunkt der Erfahrung vorangetrieben werden: in die Parallelwelt (die Heterotopie), in die Vorwelt (die Geschichte) und in die Zukunft (die Welt unserer lebensgierigen Kinder). Für Transkribisten sind alle Bilder JETZTZEIT. ${ }^{29}$

Eine Übersetzung kann niemals dieselbe Authentizität wie das Original besitzen. Der Übersetzer verwandelt den Originaltext unvermeidlich stärker, als es ein Transkribist je tun würde. Die Unschärfe ist Wort für Wort, Satz für Satz unvermeidbar, ohne dass eigentlich falsch übersetzt wird. Ortega y Gasset bezeichnet deshalb die Übersetzung als dauernden literarischen „flou“, also als Unschärfe wie bei fotografischen Aufnahmen. ${ }^{30}$ Die Authentizität des Werks wird nicht nur trotz der Fehler, sondern auch wegen ihnen bestätigt, weil das Schreiben das Fortleben in der Evolution zur Heterotopie ist. Dadurch verweist Kluge den peniblen Leser paradoxerweise in eine andere Richtung, nämlich auf sich selbst, und entlarvt ihn als scharfen Beobachter. Schließlich entsteht das unheimliche Gefühl, dass der Text oder der Autor schon auf einen solchen Leser gewartet hat und ihn in dem Moment, in dem er ihn betrachtet, zurückbeobachtet. In „JETZTZEIT“. Hier entsteht das Paradox, dass je exakter sich der Leser auf den Text konzentriert, dieser umso vager

${ }^{27}$ Vgl. Alexander Kluge, Das Bohren harter Bretter. 133 politische Geschichten, Berlin, Suhrkamp, 2011, S. 7. Die Danksagungen am Ende der neu veröffentlichten Bücher zeigen die Namen der Personen, mit denen Kluge zusammenarbeitet.

${ }^{28}$ Vgl. Claudia Buffagni et al., The Translator as Author. Perspectives on Literary Translation, Berlin, Lit Verlag, 2009.

${ }_{29}$ Alexander Kluge/Gerhard Richter, Nachricht von ruhigen Momenten, Berlin, Suhrkamp, 2013, S. 90 .

${ }^{30}$ Ortega y Gasset, Miseria, S. 23. 
und unklarer wird. In seinem Interview mit Rainer Stollmann erläutert Kluge seine Haltung gegenüber Philologen:

\begin{abstract}
Ich halte von Germanisten außerordentlich viel, nehmen Sie einmal eine Figur aus den Lebensläufen, den Pädagogen Schwebkowski, dessen Geschichte Nietzsches Definition des Philologen vorangestellt ist. Wer Bücher liebt und pflegt und immer wieder dafür sorgt, daß sie neu gelesen werden und rein bleiben von Fehlern und Irrtümern, indem er sie interpretiert, der ist Philologe. Das ist ein Panegyrikus auf diesen Beruf, und Nietzsche ist darin einer der größten Philologen. Insofern ist das Wort Philologe, „Wortliebhaber“, eine wissenschaftliche Struktur, die ich seit F. A. Wolf, also dem Begründer der Altphilologie an der Universität Göttingen im 18. Jahrhundert, aufs äußerste verehre und gleichstelle mit dem Naturwissenschaftler, der nur in der Rabiatheit, der Aggressivität seiner Forschung, davon abweicht. ${ }^{31}$
\end{abstract}

Kluge stellt hier den Philologen mit dem Naturwissenschaftler gleich, welcher, ,nur in der Rabiatheit, der Aggressivität seiner Forschung“ abweicht. Wenn der Philologe oder Übersetzer etwas von prekären, unübersetzbaren Faktoren erklären will, sieht er sich hier im Zwiespalt gefangen. Soll er korrigieren und in Übereinstimmung bringen oder die Verständlichkeit opfern? Dabei muss sich die Person aufteilen, in den Transkribisten, den Interpreten, den Übersetzer und den Kommentator, weil man nur voneinander getrennt solche Paradoxien behandeln kann. Die übersetzten Texte, die Kommentare und die allgemeine Erläuterung in der koreanischen Übersetzung von Lebensläufen können deshalb als einander beobachtende Paradoxien, als parallele Meinungen verstanden werden. Zu der Aufspaltung des Übersetzers kommen die Regeln und Forderungen des Herausgebers, des Editors und Revisors des Verlages und die Ansichten der Kollegen, die bei der Interpretation helfen, hinzu.

\title{
V.
}

Das Vorliegen verschiedener Versionen eines Werkes in der Zielsprache gewährleistet Pluralität und stellt somit den Idealfall dar, oftmals ist deren Publikation jedoch aufgrund des Buchmarktsystems und der Urheberrechte nicht früh möglich. Der Übersetzer sollte deshalb während der Übersetzung die potentielle Leserschaft im Kontext von Gesellschaft, Geschichte und Kultur lokalisieren, damit die Leser der Übersetzung zwischen ihrer Befindlichkeit und den Geschichten, die von ihrer Realität weit entfernt liegen, einen besseren Zusammenhang schaffen können.

${ }^{31}$ Alexander Kluge, Die Entstehung des Schönheitssinns aus dem Eis. Gespräch über Geschichten mit Alexander Kluge, Berlin, Kadmos, 2005, S.40. Das Zitat von Nietzsche kommt, entgegen Kluges Aussage, nicht in der Geschichte von Schwebkowski, sondern in einer anderen Geschichte in Lebensläufe, „Der Pädagoge von Klopau“, vor. Diesen kann man in dem Sinne als eine Gegenfigur zu dem Gelehrten Schincke verstehen, dass er seine Schüler für seine Macht opfert. Das Zitat stammt aus einem Paragraphen von Die Fröhliche Wissenschaft. Dabei geht es wiederum um die „Arbeit in usum Delphinorum“, d.h. sowohl um die Macht der Zukunft, als auch um den Schutz des Schülers und um die Zensur oder den Kommentar der Philologen. Vgl. Friedrich Nietzsche, Die Fröhliche Wissenschaft, in Sämtliche Werke (Kritische Studienausgabe), Bd. 3, München, dtv, Berlin/New York, Walter de Gruyter, 1988, S. 458f., § 102. 
Übersetzung wird in diesem Sinne zu einer Art politischer Fragestellung, bei der der Übersetzer zu entscheiden hat, in welchem Ausmaß die verdeckten historischen Tatsachen als solche entlarvt werden sollen und inwieweit er es den Lesern, ihrem Wissen, ihren Vorstellungen und Forschungen überlassen soll, den Konnex selbst herzustellen. Der Übersetzer gerät in eine ähnliche Situation wie der ursprüngliche Verfasser, der einst justierte, unabhängig von seinem Erfolg, bis zu welchem Grad er dem Leser die Hoheit über die Interpretation ließ. Diese babylonische Hybris ist jedoch sachgemäß zum Scheitern verurteilt, denn Worte haben die Tendenz, auseinander zu brechen. ${ }^{32}$

Der kindliche Wunsch des Übersetzers könnte nachträglich wie eine „Ruine“, eine „Erinnerung“ ${ }^{33}$ erscheinen und auch als einer der Hintergründe für die Übersetzung angeführt werden. Wir könnten uns vielleicht der Aussage von Kluge selbst über seine Autorschaft für die Erklärung der Übersetzung bedienen. Kluge zitiert Henri Lefebvre in seiner Ulmer Dramaturgie:

Zwischen Bedürfnis und Wunsch gibt es viele Vermittlungen. Genaugenommen liegt alles dazwischen: die gesamte Gesellschaft (Produktionstätigkeiten und Konsumtionsweisen), die Kultur, Vergangenheit und Geschichte, die Sprache, die Normen, die Befehle und Verbote, die Hierarchie der Werte und Neigungen. ${ }^{34}$

Als der gesellschaftliche Grund für die späte Übersetzung könnte der Boom der Weltliteratur auf dem heutigen koreanischen Buchmarkt angegeben werden. Das Bildungskonzept der „Weltliteratur“" selbst ist ein aus Preußen von den Japanern „,importierter“ und durch deren Kolonialherrschaft nach Korea gebrachter Begriff, der ursprünglich aus den Gesprächen zwischen Goethe und Eckermann stammt. Wenn die „Weltliteratur“" mit ihrem klassischen Kanon früher global als kolonialistische Propaganda der westlichen Welt fungierte und lokal zur Rechtfertigung von Modernisierung und „Verwestlichung“ diente, war die Situation für eine authentische Weltliteratur nicht ganz „stimmig“، 35

So gilt das neue Weltliteratur-Konzept sozusagen als Kommentar und Kritik zur „Originalkultur“. Wie Kluge sagt, ist „Kritik [...] kein bloßes Schriftgut, kein Rechthaben in Form von Büchern gegenüber anderen Büchern, sondern konsequente aktive Reparaturarbeit“. ${ }^{36}$ Kluges Vorwort $\mathrm{zu}$ den Lebensläufen

\footnotetext{
${ }^{32}$ Vgl. Jacques Derrida, „Babylonische Türme. Wege, Umwege, Abwege“, in Übersetzung und Dekonstruktion, Frankfurt a. M., Suhrkamp, 1997, S. 119ff.

33 Klaus Eder/Alexander Kluge, Ulmer Dramaturgien, Reibungsverluste, München/Wien, Carl Hanser Verlag, 1980, S. 93.

34 Ibid., S. 94.

${ }^{35}$ Vgl. Douglas Robinson, Translation and Empire. Postcolonial Theories Explained, Manchester, St. Jerome Publishing, 1997; 김영희, 유희석 (엮음), 세계문학론. 지구화시대 문학의 쟁점들, 서울, 창비, 2009 (Younghee Kim, Huisok Yoo (Hrsg.), Diskurse der Weltiteratur. Streitpunkte der Literatur in der globalisierten Zeit, Seoul, Changbi Publishers, 2009); 조영일, 세계문학의 구조, 서울, 도서출판 b, 2011 (Young-il Cho, Die Struktur der Weltiteratur, Seoul, b-books, 2011); 박숙자, 속물 교양의 탄생. 명작이라는 식민의 유령, 서울, 푸른역사, 2012 (Sukja Park, Die Geburt der Snob-Bildung. Ein Gespenst des Kolonialismus namens Meisterwerk, Seoul, blue history, 2012); Alexander Kluge/Oskar Negt, Öffentlichkeit und Erfahrung. Zur Organisationsanalyse von bürgerlicher und proletarischer Öffentlichkeit, Frankfurt a. M., Suhrkamp, 1972, S. 83f.

${ }^{36}$ Kluge, Personen und Reden, S. 70.
} 
beginnt folgendermaßen: „Die Erzählungen dieses Bandes stellen aus sehr verschiedenen Aspekten die Frage nach der Tradition. “ ${ }^{37}$ Dabei handelt es sich nicht nur um Kritik an der damaligen deutschen Gesellschaft sondern auch um Kritik an dem Literaturkonzept im Allgemeinen. Die Übersetzung von Alexander Kluges Lebensläufen selbst könnte als Kommentar zu all dem dienen.

Die neue Bildung wäre „Anpassung und Widerstand““38, wie Kluge mit Hellmut Becker sagt. Bildung ist bei Kluge nicht „etwas in einen geformten Zustand [zu] bringen“ ${ }^{39}$. Sie sei „Hilfestellung“40 um den schneckenähnlichen, kopfscheuen Intellektuellen „zu locken, seinen Mut zu stärken“41. Mit Immanuel Kant sagt Kluge, es sei bei der Bildung „nach authentischer Lebendigkeit [...] zu suchen“42. Es gehe um „Einfühlung ${ }^{\star 43}$.

Die Übersetzung fungiert für mich als ein Lehrprozess, eine Übung zur Einfühlung. Walter Benjamin sagt: „Kommentare und Übersetzungen verhalten sich zum Text wie Stil und Mimesis zur Natur: dasselbe Phänomen unter verschiedenen Betrachtungsweisen“"44. All die Paradoxien zwischen den Texten eines Buches richten sich auf Authentizität. Kluges „engagierter Pluralismus“45 wird damit auch zur Aufgabe des Übersetzers, zur Anweisung an den Transkribisten und zum Anliegen des Kommentators.

${ }^{37}$ LL, S. 7.

${ }^{38}$ Kluge, Das fünfte Buch, S. 502.

${ }^{39}$ Kluge, Personen und Reden, S. 73.

${ }^{40}$ Ibid.

${ }^{41}$ Ibid

${ }^{42}$ Ibid

${ }^{43}$ Ibid., S. 74.

${ }^{44}$ Walter Benjamin, „Diese Anpflanzungen sind dem Schutze des Publikums empfohlen“, in Einbahnstraße, in Gesammelte Werke, Bd. 1, Frankfurt a. M., Zweitausendeins, 2011, S. 964.

${ }^{45}$ Müller, „Verwendungsweisen“, S. 56. 
